\title{
Prognostic Value of Major Cardiac Event Risk Score Estimated With Gated Myocardial Perfusion Imaging in Japanese Patients With Coronary Artery Disease
}

\author{
Shunichi Yoda, ${ }^{1} \mathrm{MD}$, Kanae NaKAnishI, ${ }^{1} \mathrm{MD}$, Ayako TANo,${ }^{1} \mathrm{MD}$, Yusuke HorI,${ }^{1} \mathrm{MD}$, \\ Misa Hayase, ${ }^{1}$ MD, Takashi MineKI, ${ }^{1}$ MD, Yasuyuki Suzuki, ${ }^{1}$ MD, \\ Naoya Matsumoto, ${ }^{1} \mathrm{MD}$, and Atsushi Hirayama, ${ }^{1} \mathrm{MD}$
}

\section{SUMMARY}

We published a cardiac event risk score (CERS) predicting the risk of major cardiac events (MCEs) within 3 years. The purpose of this study was to verify the prognostic value of the CERS before and after treatment in Japanese patients with coronary artery disease.

We retrospectively investigated 612 patients who underwent rest ${ }^{201} \mathrm{Tl}$ and stress ${ }^{99 \mathrm{~m}} \mathrm{Tc}$-tetrofosmin myocardial perfusion single photon emission computed tomography (SPECT) between October 2004 and March 2013 and who had a significant stenosis with $\geq 75 \%$ narrowing of the arterial diameter detected by coronary angiography performed after confirmation of $\geq 5 \%$ ischemia with the SPECT. The patients underwent treatment including revascularization and medication, and thereafter, were re-evaluated with SPECT during a chronic phase and followed-up to confirm prognosis for $\geq$ 1 year. The endpoint was the onset of MCEs during the follow-up.

During the follow-up $(36.7 \pm 14.5$ months), 50 patients $(8.7 \%)$ experienced MCEs comprising cardiac death $(n=$ $16)$, non-fatal myocardial infarction $(n=4)$, and unstable angina pectoris $(n=30)$. The multivariate Cox proportional hazards regression model analysis for the actual occurrence of MCEs showed the summed difference score \% and MCE risks estimated with the CERS after treatment to be significant independent variables. Ischemic reduction after treatment contributed significantly to a decrease in the MCE risks. The MCE risks estimated with the CERS after treatment were generally consistent with the incidence of the MCEs actually observed.

The CERS after treatment is a valuable formula for predicting prognosis in Japanese patients with coronary artery disease. (Int Heart J 2016; 57: 408-416)

Key words: Prognosis, Risk stratification, Risk calculator, Ischemic heart disease, SPECT

$\mathrm{P}$ rediction of a cardiac event risk is important for decisions concerning an optimal therapeutic strategy in patients with coronary artery disease (CAD). A wellknown cardiac event risk score is a prediction algorithm developed in an epidemiological study (Framingham study) in the United States. ${ }^{1)}$ The algorithm, which is a valuable tool to predict the risk of cardiac events in the 10 subsequent years in Caucasians, is highly recommended in the Guideline on the Assessment of Cardiovascular Risk published by the American College of Cardiology and American Heart Association in 2013. ${ }^{2}$

Myocardial perfusion single photon emission computed tomography (SPECT) is widely recognized as a useful imaging methodology for the prediction of future cardiac events in patients with known or suspected CAD since the reports of Hachamovitch, et al. ${ }^{3,4)}$ Also, in Japan, myocardial perfusion SPECT has commonly been used to predict cardiac events in patients with CAD. Risk stratification of cardiac events by nuclear cardiology has been demonstrated in some large-scale prognostic studies including the multicenter prospective Japanese Assessment of Cardiac Events and Survival Study in patients with ischemic heart disease (J-ACCESS), in asymptomatic patients with type 2 diabetes (J-ACCESS 2), ${ }^{6}$ in patients with chronic kidney disease (J-ACCESS 3), ${ }^{7}$ and in another single-center large-scale prospective study. ${ }^{8)}$

Nakajima, et $a l^{9)}$ published a Heart Risk Table, which is the first cardiac event risk score predicting the risk of major cardiac events (MCEs) in Japanese patients with CAD within 3 years, prepared on the basis of predictors obtained from the J-ACCESS database $(n=2,395)$. MCEs predicted with the Heart Risk Table, however, include severe heart failure unlike MCEs defined in non-Japanese studies. ${ }^{3,4)}$

We, therefore, conducted a single-center large-scale retrospective prognostic study in 2,579 Japanese patients with CAD

From the ${ }^{1}$ Department of Cardiology, Nihon University School of Medicine, Tokyo, Japan.

Address for correspondence: Shunichi Yoda, MD, Department of Cardiology, Nihon University School of Medicine, 30-1 Oyaguchi-Kamicho, Itabashi-ku, Tokyo 1738610, Japan. E-mail: masteryoda@mf.point.ne.jp

Received for publication November 1, 2015. Revised and accepted January 5, 2016

Released in advance online on J-STAGE June 29, 2016.

All rights reserved by the International Heart Journal Association. 
undergoing nuclear cardiology to prepare a cardiac event risk score (CERS) predicting the risk of MCEs excluding severe heart failure within 3 years. ${ }^{10)}$ In the present study, we attempted to verify the prognostic values of the CERS before and after treatment in Japanese patients with CAD.

\section{MeThodS}

The Institutional Review Board of Nihon University Itabashi Hospital approved this study, which proceeded in accordance with the ethical standards established in the 1964 Declaration of Helsinki. All study participants provided written informed consent prior to inclusion in the study.

Patient population: We retrospectively investigated 612 patients with CAD who underwent rest ${ }^{201} \mathrm{Tl}$ and stress ${ }^{99 \mathrm{~m}} \mathrm{Tc}$ tetrofosmin myocardial perfusion SPECT ${ }^{8,10-16)}$ at Nihon University Itabashi Hospital between October 2004 and March 2013 and who had a significant stenosis with $75 \%$ or greater narrowing of the arterial diameter detected by coronary angiography (CAG) performed after confirmation of at least 5\% ischemia by the SPECT. The patients underwent treatment including revascularization and medication, and thereafter, were re-evaluated with the SPECT during a chronic phase and followed up to confirm prognosis for at least one year. The interval between the first SPECT and the CAG was $1.6 \pm 3.2$ months, that between the $\mathrm{CAG}$ and revascularization was $0.8 \pm$ 3.9 months, and that between the revascularization and the second SPECT was $8.3 \pm 8.2$ months. The second SPECT was performed at $13.3 \pm 10.6$ months after the first SPECT. We excluded patients aged $\leq 20$ years, as well as those with hypertrophic or dilated cardiomyopathy, with serious valvular heart disease, with heart failure with class III or higher New York Heart Association (NYHA) functional classification, and with less than $5 \%$ ischemia detected by the first SPECT. Patients were diagnosed as hypertensive if they had systolic pressure $>$ $140 \mathrm{mmHg}$ and/or diastolic pressure $>90 \mathrm{mmHg}$ within the past 3 months or were currently under medication to lower blood pressure; as having diabetes mellitus if they had fasting blood glucose $>126 \mathrm{mg} / \mathrm{dL}$ and/or casual blood glucose $>200$ $\mathrm{mg} / \mathrm{dL}$ within the past 3 months or were under medication to control hyperglycemia, or were already diagnosed with diabetes mellitus; as having hyperlipidemia if they had low-density lipoprotein cholesterol $>140 \mathrm{mg} / \mathrm{dL}$ within the past 3 months or were currently under medication to normalize hypercholesterolemia; as having typical chest pain if they had substernal chest pain or discomfort that was provoked by exertion or emotional stress and was relieved by rest and/or nitroglycerin. Follow-up examinations were based on medical records for patients who periodically attended the hospital and responded to a mailed questionnaire for patients who did not attend. The follow-up was successful for 572 patients (93\%) but unsuccessful for the remaining 40 patients. Data from all 572 patients were finally included in the analysis. All 612 patients enrolled in this study were a different population from the patients $(n=2,579)$ enrolled in the previous study conducted to prepare the CERS. ${ }^{10)}$

ECG-gated dual-isotope myocardial perfusion SPECT: The rest ${ }^{201} \mathrm{Tl}$ and stress ${ }^{99 \mathrm{~m}} \mathrm{Tc}$-tetrofosmin ECG-gated myocardial perfusion SPECT procedure was performed according to a previously reported protocol. ${ }^{8,10-16)}$ All patients received an in- travenous injection of ${ }^{201} \mathrm{Tl}(111 \mathrm{MBq})$ and a 16-frame gated SPECT image was initiated 10 minutes after the injection during rest. Next, an intravenous injection of ${ }^{99 \mathrm{~m}} \mathrm{Tc}$-tetrofosmin (740 MBq) was administered under stress induced by ergometer exercise in $23 \%$ of the patients or by adenosine triphosphate in $77 \%$ of the patients. Sixteen-frame gated SPECT image acquisition was initiated 30 minutes after the exercise or 30 to 60 minutes after the adenosine triphosphate stress. The acquisition was performed with the patient in a supine position and then in a prone position. No attenuation or scatter correction was used. A 12-lead ECG was monitored continuously during the stress tests. Heart rate and blood pressure were recorded at baseline and every minute for at least 3 minutes after the stress.

The projection data over $360^{\circ}$ were obtained with $64 \times 64$ matrices and a circular orbit. A triple-detector SPECT system equipped with low-energy high-resolution collimators was used (Toshiba, GCA9300A, Tokyo). SPECT images were reconstructed from the data with a data processor (Philips North America, JETStream Workspace 3.0, MA, USA) combined with Butterworth filters of ${ }^{201} \mathrm{Tl}$ (order 5; cut-off frequency 0.42 cycles $/ \mathrm{cm}$ ) and ${ }^{99 \mathrm{~m}} \mathrm{Tc}$ (order 5 ; cut-off frequency 0.44 cycles/ $\mathrm{cm})$ and a ramp filter.

SPECT image interpretation: The SPECT images were divided into 20 segments $^{12)}$ on 3 short-axis (distal, mid, basal) and one vertical long-axis (mid) slices, and the tracer uptake of each segment was visually scored using a 5-point scale (0: normal; 1: slight reduction of uptake; 2 : moderate reduction of uptake; 3: severe reduction of uptake; and 4: absence of uptake). The sum total of the scores of 20 segments in the stress and rest images provided the summed stress score (SSS) and the summed rest score (SRS), respectively. The summed difference score (SDS) was calculated as the difference between the SSS and SRS. The respective summed scores were converted to percent of the total myocardium (visual \% myocardium). Visual $\%$ myocardium was derived from a summed score divided by the maximum potential score $(4 \times 20)$ and multiplied by 100 . When the SSS score was 4 , the visual $\%$ myocardium was $5 \%$. A change in $\operatorname{SDS} \%(\triangle \mathrm{SDS} \%)$ was a difference between SDS\% estimated by the first and second SPECT. The visual semi-quantitative scoring was performed by two independent expert interpreters who were not aware of any clinical information. Cohen's kappa $(\kappa)$, which was calculated to determine the inter-observer variability for the summed defect score, was 0.92 , indicating very good reproducibility. Sixteen-frame quantitative gated SPECT data were analyzed with QGS ${ }^{\mathrm{TM}}$ software (Cedars-Sinai Medical Center, Los Angeles, CA, USA) to calculate the left ventricular ejection fraction (LVEF, \%), end-diastolic volume (LVEDV, mL), and endsystolic volume (LVESV, $\mathrm{mL}$ ) as described by Germano, et al. $^{17)}$

Evaluation of eGFR: The estimated glomerular filtration rate (eGFR) was calculated from serum creatinine levels at the time of SPECT for each patient with the Modification of Diet in Renal Disease Equation for Japanese Patients proposed by the Japanese Society of Nephrology ${ }^{18)}$ as follows:

$\mathrm{eGFR}=\mathrm{A} \times 194 \times(\text { Serum Creatinine })^{-1.094} \times(\text { Age })^{-0.287}$, where $\mathrm{A}$ is 1 for men and 0.739 for women and eGFR is expressed as $\mathrm{mL} /$ minute $/ 1.73 \mathrm{~m}^{2}$.

Patient follow-up: The study endpoint was the onset of MCEs during the follow-up, consisting of cardiac death, non-fatal 
Table I. Characteristics of Patients With and Without Major Cardiac Events

\begin{tabular}{lccccc}
\hline & Major cardiac event (+) & \multicolumn{3}{c}{ Major cardiac event (-) } \\
& $n=50$ & & $n=522$ & $P$ \\
\hline Male gender & 42 & $84 \%$ & 422 & $81 \%$ & 0.7220 \\
Age & $68 \pm 12$ & & $67 \pm 9$ & & 0.4424 \\
Typical chest pain & 18 & $36 \%$ & 195 & $37 \%$ & 0.9710 \\
History of MI & 23 & $46 \%$ & 195 & $37 \%$ & 0.2938 \\
History of revascularization & 32 & $64 \%$ & 244 & $47 \%$ & 0.0289 \\
Hypertension & 40 & $80 \%$ & 414 & $79 \%$ & 0.9459 \\
Diabetics & 31 & $62 \%$ & 239 & $46 \%$ & 0.0408 \\
Hyperlipidemia & 29 & $58 \%$ & 391 & $75 \%$ & 0.0156 \\
Smoking & 19 & $38 \%$ & 196 & $38 \%$ & 0.9285 \\
Aspirin & 48 & $96 \%$ & 503 & $96 \%$ & 0.9569 \\
Statins & 23 & $46 \%$ & 323 & $62 \%$ & 0.0411 \\
$\beta$-blockers & 18 & $36 \%$ & 205 & $39 \%$ & 0.7631 \\
Ca-antagonists & 24 & $48 \%$ & 246 & $47 \%$ & 0.9760 \\
Nitrates & 17 & $34 \%$ & 204 & $39 \%$ & 0.5804 \\
ARB & 24 & $48 \%$ & 255 & $49 \%$ & 0.9736 \\
ACE & 9 & $18 \%$ & 49 & $9 \%$ & 0.0925 \\
eGFR & $57.3 \pm 26.3$ & & $61.4 \pm 23.4$ & & 0.2488 \\
Angiographic CAD & & & & & \\
1 Vessel & 12 & $24 \%$ & 176 & $34 \%$ & 0.2151 \\
2 Vessels & 20 & $40 \%$ & 169 & $33 \%$ & 0.3485 \\
3 Vessels & 18 & $36 \%$ & 177 & $34 \%$ & 0.8092 \\
Treatment & & & & & \\
PCI & 38 & $76 \%$ & 395 & $76 \%$ & 0.9039 \\
$\quad$ POBA & $(2)$ & $(5 \%)$ & $(15)$ & $(4 \%)$ & 0.9944 \\
BMS & $(7)$ & $(18 \%)$ & $(79)$ & $(20 \%)$ & 0.9839 \\
$\quad$ DES & $(29)$ & $(76 \%)$ & $(301)$ & $(76 \%)$ & 0.8542 \\
CABG & 1 & $2 \%$ & 44 & $8 \%$ & 0.1808 \\
Medication & 11 & $22 \%$ & 83 & $16 \%$ & 0.3617 \\
\hline
\end{tabular}

MI indicates myocardial infarction; ARB, angiotensin receptor blocker; ACE, angiotensin converting enzyme; eGFR, estimated glomerular filtration rate; CAD, coronary artery disease; PCI, percutaneous coronary intervention; POBA, plain old balloon angioplasty; BMS, bare-metal stent; DES, drug-eluting stent; and CABG, coronary artery bypass grafting.

myocardial infarction (MI), and unstable angina pectoris (UAP) identified from medical records or from responses to a mailed out questionnaire. A diagnosis of UAP was provided for a patient who required unscheduled hospitalization for the management of UAP, occurring within 24 hours of the most recent symptoms, and who had worsening ischemic discomfort, objective evidence of myocardial ischemia, and negative cardiac biomarkers. All 572 patients were followed up for at least one year $(36.7 \pm 14.5$ months $)$ after the second SPECT. When a patient had multiple MCEs, only the first event was taken as the study endpoint.

Estimated MCE risks within 3 years with the CERS: MCE risk (\%) within 3 years before and after treatment was estimated with the following formula ${ }^{10)}$ predicting the risk of MCEs except severe heart failure on the basis of 4 predictors consisting of age, diabetes mellitus (DM), SSS, and eGFR:

$$
\begin{aligned}
& \text { MCE risk }(\% / 3 \text { years })=1 /\{1+\operatorname{Exp}[-(-3.176+0.018 \times \\
& \text { age }+0.602 \times \mathrm{DM}-0.022 \times \mathrm{eGFR}+0.051 \times \mathrm{SSS})]\} \times \\
& 100
\end{aligned}
$$

where age is in years, DM is 1 for presence or 0 for absence, eGFR is $\mathrm{mL} / \mathrm{minute} / 1.73 \mathrm{~m}^{2}$, and SSS is continuous variables. Estimated MCE risk reduction (\%) was defined as a difference in MCE risk (\%) between before and after treatment.

Statistical analysis: Continuous variables were calculated as the mean and standard deviation. Intergroup comparisons of continuous and categorical variables were achieved with the unpaired $t$ test and the chi-square test, respectively. The paired $t$ test was used to compare MCE risks estimated before and af- ter treatment. Univariate analyses used the Cox proportional hazards regression model and multivariate analyses the stepwise Cox proportional hazards regression model. Linear regression analysis of $\triangle \mathrm{SDS} \%$ as an independent variable and estimated MCE risk reduction within 3 years with the CERS as a dependent variable was performed by the least squares method to calculate a correlation coefficient $(r)$ suggesting a relationship between the two variables. Kaplan-Meier survival analysis was used to compare between MCE risks estimated with the CERS after treatment and actual MCE risks. The chisquare for trend test was used to detect any differences between 3 categories divided by tertile. All data were analyzed with MedCalc Software Version 13.1.2.0 (Mariakerke, Belgium). A $P$ value of $<0.05$ was considered statistically significant.

\section{RESULTS}

Major cardiac event rates and patient characteristics: During the follow-up, 50 (8.7\%) of 572 patients experienced MCEs including cardiac death $(n=16)$, non-fatal MI $(n=4)$, and UAP $(n=30)$. CAG, which was performed in all 34 patients with non-fatal MI and UAP, indicated severe stenosis. Of those, 33 patients underwent percutaneous coronary intervention (PCI) for culprit lesions and the remaining patient underwent coronary artery bypass grafting $(\mathrm{CABG})$. Major causes of UAP were progression of residual non-culprit stenosis $(n=$ 
Table II. Summary of Visual \% Myocardium, Ischemic Reduction, Left Ventricular Ejection Fraction and Volume, and MCE Risk Estimated With the Cardiac Event Risk Score

\begin{tabular}{|c|c|c|c|}
\hline & $\begin{array}{l}\text { Major cardiac event }(+) \\
\quad n=50\end{array}$ & $\begin{array}{l}\text { Major cardiac event (-) } \\
\qquad n=522\end{array}$ & $P$ \\
\hline SSS\% before treatment & $18.3 \pm 11.9$ & $19.0 \pm 11.0$ & 0.6510 \\
\hline SRS\% before treatment & $6.3 \pm 8.3$ & $5.0 \pm 7.9$ & 0.2721 \\
\hline SDS\% before treatment & $12.0 \pm 6.3$ & $14.0 \pm 7.6$ & 0.0673 \\
\hline SSS\% after treatment & $14.9 \pm 12.4$ & $10.9 \pm 11.4$ & 0.0200 \\
\hline SRS\% after treatment & $7.5 \pm 10.9$ & $5.3 \pm 8.8$ & 0.0948 \\
\hline SDS \% after treatment & $7.4 \pm 7.7$ & $5.6 \pm 6.3$ & 0.0700 \\
\hline$\Delta \mathrm{SDS} \%$ & $4.6 \pm 7.9$ & $8.4 \pm 9.0$ & 0.0044 \\
\hline $5 \% \geq$ ischemic reduction & $23 \quad 46 \%$ & $353 \quad 68 \%$ & 0.0035 \\
\hline Rest LVEF before treatment & $52.5 \pm 15.5$ & $57.2 \pm 14.2$ & 0.0262 \\
\hline Rest LVEDV before treatment & $103.4 \pm 59.8$ & $92.3 \pm 42.8$ & 0.0923 \\
\hline Rest LVESV before treatment & $56.8 \pm 54.4$ & $44.3 \pm 36.5$ & 0.0284 \\
\hline Stress LVEF before treatment & $50.1 \pm 14.6$ & $54.7 \pm 13.8$ & 0.0242 \\
\hline Stress LVEDV before treatment & $122.7 \pm 60.6$ & $109.3 \pm 47.3$ & 0.0631 \\
\hline Stress LVESV before treatment & $69.1 \pm 55.2$ & $54.9 \pm 41.0$ & 0.0236 \\
\hline Rest LVEF after treatment & $52.9 \pm 15.8$ & $59.2 \pm 13.9$ & 0.0024 \\
\hline Rest LVEDV after treatment & $104.1 \pm 56.7$ & $89.2 \pm 40.4$ & 0.0173 \\
\hline Rest LVESV after treatment & $56.8 \pm 54.4$ & $41.0 \pm 34.3$ & 0.0035 \\
\hline Stress LVEF after treatment & $50.3 \pm 15.2$ & $57.8 \pm 13.6$ & 0.0002 \\
\hline Stress LVEDV after treatment & $121.2 \pm 60.6$ & $103.3 \pm 45.4$ & 0.0103 \\
\hline Stress LVESV after treatment & $68.7 \pm 57.7$ & $48.5 \pm 38.0$ & 0.0007 \\
\hline Estimated MCE risk (\%/3 years) with CERS before treatment & $14.1 \pm 11.1$ & $11.5 \pm 8.6$ & 0.0417 \\
\hline Estimated MCE risk (\%/3 years) with CERS after treatment & $13.9 \pm 10.8$ & $9.4 \pm 8.0$ & 0.0003 \\
\hline Estimated MCE risk reduction (\%/3 years) with CERS & $0.2 \pm 6.3$ & $2.0 \pm 4.7$ & 0.0126 \\
\hline
\end{tabular}

The treatment mentioned in this table consists of percutaneous coronary intervention, coronary artery bypass grafting, and medication. SSS indicates summed stress score; SRS, summed rest score; SDS, summed difference score; $\triangle$ SDS\%, the difference in SDS\% between the first and second SPECT; LVEF, left ventricular ejection fraction; LVEDV, left ventricular ejection end-diastolic volume; LVESV, left ventricular ejection end-systolic volume; MCE, major cardiac event; and CERS, cardiac event risk score.

13), new stenotic lesions $(n=3)$, re-stenosis at the revascularization site $(n=3)$, progression of stenosis at regions adjacent to the revascularization site $(n=6)$, and progression of stenosis at jailed branches of the revascularization site $(n=5)$.

Table I summarizes the characteristics of the patients with and without MCEs. Mean age of the patients with and without MCEs was 68 and 67 years old, respectively, and $84 \%$ and $81 \%$ of the patients with and without MCEs were males. The proportions of patients with typical chest pain, a history of MI, hypertension, or a smoking habit were not significantly different between the two groups with and without MCEs. The proportions of patients with a history of revascularization and those with DM were significantly higher in the group with than without MCEs $(P<0.041)$. The proportions of patients with hyperlipidemia and those treated with statins were significantly lower in the group with than without MCEs $(P<0.042)$. The proportions of patients treated with other medications including aspirin, $\beta$-blockers, calcium antagonists, nitrates, angiotensin II receptor blockers, or angiotensin converting enzyme inhibitors were not significantly different between the two groups. The eGFR of patients with MCEs was $57.3 \pm 26.3 \mathrm{~mL} /$ minute $/ 1.73 \mathrm{~m}^{2}$, which was similar to that of patients without MCEs $\left(61.4 \pm 23.4 \mathrm{~mL} /\right.$ minute $\left./ 1.73 \mathrm{~m}^{2}\right)$. The severity of CAD detected by CAG was similar between the two groups. The proportion of patients undergoing PCI was 76\% (38/50) in the group with MCEs and 76\% (395/522) in the group without MCEs. As a breakdown of PCI, the proportions of patients treated with plain old balloon angioplasty were $5 \%(2 / 38)$ in the group with MCEs and 4\% (15/395) in the group without MCEs; those treated with a bare-metal stent were $18 \%(7 / 38)$ in the group with MCEs and 20\% (79/395) in the group without MCEs; those treated with a drug-eluting stent were $76 \%$ (29/38) in the group with MCEs and 76\% (301/395) in the group without MCEs. One of the patients with MCEs and 44 of the patients without MCEs underwent CABG. The remaining patients (11 and 83 in the groups with and without MCEs, respectively) received medication alone, mainly due to an inappropriate lesion for revascularization.

Visual \% myocardium, left ventricular function, and estimated MCE risks: Table II summarizes visual \% myocardium (SSS\%, SRS\% or SDS\%) for myocardial defects detected by the SPECT as well as LVEF, LVEDV, and LVESV estimated with the quantitative gated SPECT before and after the treatment in the patients with and without MCEs; $\triangle \mathrm{SDS} \%$ and the number of patients having $\geq 5 \%$ ischemic reduction in patients with and without MCEs; MCE risks estimated with CERS before and after the treatment in the patients with and without MCEs. The visual \% myocardium before the treatment was not different between the patients with and without MCEs. After the treatment, the SSS\% was significantly higher in the patients with than without MCEs $(14.9 \pm 12.4$ versus $10.9 \pm 11.4$; $P=0.0200)$. The $\Delta \mathrm{SDS} \%$ was significantly higher in the patients without than with MCEs $(8.4 \pm 9.0$ versus $4.6 \pm 7.9 ; P=$ $0.0044)$. The proportions of patients having $\geq 5 \%$ ischemic reduction after the treatment were $46 \%$ and $68 \%$ in the patients with and without MCEs, respectively $(P=0.0035)$. The rest and stress LVEF were generally higher while the rest and stress LVEDV and LVESV were smaller in the patients without than with MCEs, irrespective of whether it was estimated before or after the treatment. The MCE risk within 3 years estimated 
Table III. Univariate Cox Proportional Hazards Regression Analysis

\begin{tabular}{|c|c|c|c|c|}
\hline & Chi-Square & Hazard ratio & $\begin{array}{c}95 \% \\
\text { Confidence interval }\end{array}$ & $P$ \\
\hline Age & 0.9739 & 1.0156 & $0.9850-1.0472$ & 0.3237 \\
\hline Male sex & 0.3513 & 1.2569 & $0.5924-2.6669$ & 0.5534 \\
\hline History of MI & 1.1904 & 1.3629 & $0.7837-2.3704$ & 0.2752 \\
\hline History of revascularization & 5.0678 & 1.9415 & $1.0928-3.4492$ & 0.0244 \\
\hline Hypertension & 0.0313 & 1.0647 & $0.5342-2.1217$ & 0.8594 \\
\hline Diabetes mellitus & 4.2452 & 1.8228 & $1.0327-3.2175$ & 0.0394 \\
\hline Hyperlipidemia & 5.4910 & 0.5129 & $0.2942-0.8940$ & 0.0191 \\
\hline Statin use & 4.6018 & 0.5440 & $0.3128-0.9461$ & 0.0319 \\
\hline eGFR & 1.8697 & 0.9922 & $0.9812-1.0033$ & 0.1715 \\
\hline SSS $\%$ before treatment & 0.2783 & 0.9930 & $0.9677-1.0190$ & 0.5978 \\
\hline SRS\% before treatment & 1.0506 & 1.0161 & $0.9857-1.0474$ & 0.3054 \\
\hline SDS\% before treatment & 3.4762 & 0.9579 & $0.9157-1.0020$ & 0.0623 \\
\hline SSS\% after treatment & 5.5894 & 1.0242 & $1.0042-1.0447$ & 0.0181 \\
\hline SRS\% after treatment & 3.0708 & 1.0225 & $0.9975-1.0481$ & 0.0797 \\
\hline SDS\% after treatment & 3.4063 & 1.0355 & $0.9980-1.0743$ & 0.0649 \\
\hline$\Delta \mathrm{SDS} \%$ & 8.6103 & 0.9465 & $0.9125-0.9817$ & 0.0033 \\
\hline $5 \% \geq$ ischemic reduction & 4.7610 & 0.5217 & $0.2916-0.9332$ & 0.0291 \\
\hline Rest LVEF after treatment & 9.3817 & 0.9734 & $0.9569-0.9903$ & 0.0022 \\
\hline Rest LVEDV after treatment & 5.5690 & 1.0060 & $1.0010-1.0110$ & 0.0183 \\
\hline Rest LVESV after treatment & 8.2202 & 1.0075 & $1.0024-1.0126$ & 0.0041 \\
\hline Stress LVEF after treatment & 13.7102 & 0.9664 & $0.9492-0.9840$ & 0.0002 \\
\hline Stress LVEDV after treatment & 6.5929 & 1.0059 & $1.0014-1.0104$ & 0.0102 \\
\hline Stress LVESV after treatment & 11.0891 & 1.0078 & $1.0032-1.0124$ & 0.0009 \\
\hline Estimated MCE risk (\%/3 years) with CERS before treatment & 5.0420 & 1.0291 & $1.0038-1.0550$ & 0.0247 \\
\hline Estimated MCE risk (\%/3 years) with CERS after treatment & 15.5913 & 1.0456 & $1.0228-1.0689$ & 0.0001 \\
\hline Estimated MCE risk reduction (\%/3 years) with CERS & 8.3334 & 0.9363 & $0.8955-0.9789$ & 0.0039 \\
\hline
\end{tabular}

The treatment mentioned in this table consists of percutaneous coronary intervention, coronary artery bypass grafting, and medication. MI indicates myocardial infarction; eGFR, estimated glomerular filtration rate; SSS, summed stress score; SRS, summed rest score; SDS, summed difference score; $\triangle \mathrm{SDS} \%$, the difference in SDS\% between the first and second SPECT; LVEF, left ventricular ejection fraction; LVEDV, left ventricular end-diastolic volume; LVESV, left ventricular end-systolic volume; MCE, major cardiac event; and CERS, cardiac event risk score.

Table IV. Multivariate Cox Proportional Hazards Regression Analysis

\begin{tabular}{lccc}
\hline & Hazard ratio & 95\% Confidence interval & $P$ \\
\hline$\Delta$ SDS\% & 0.9559 & $0.9559-0.9917$ & 0.0167 \\
Estimated MCE risk (\%) with CERS after treatment & 1.0399 & $1.0160-1.0644$ & 0.0010 \\
\hline
\end{tabular}

The treatment mentioned in this table consists of percutaneous coronary intervention, coronary artery bypass grafting and medication. SDS indicates summed difference score; $\triangle \mathrm{SDS} \%$, a difference between SDS\% by the first and second SPECT; MCE, major cardiac event; and CERS, cardiac event risk score.

with the CERS was significantly higher in the patients with than without MCEs, irrespective of whether it was estimated before or after the treatment. The MCE risk reduction within 3 years estimated with the CERS was significantly lower in the patients with than without MCEs $(0.2 \pm 6.3$ versus $2.0 \pm 4.7 ; P$ $=0.0126$ ).

Prediction of MCEs: The results of univariate Cox proportional hazards regression analysis showed a history of revascularization, DM, hyperlipidemia, statin use, SSS\% after treatment, $\Delta \mathrm{SDS} \%, 5 \% \geq$ ischemic reduction, left ventricular function after treatment, MCE risks estimated with the CERS before and after treatment, and MCE risk reduction within 3 years estimated with the CERS to be significant predictors for MCEs (Table III). Among these variables, $\triangle \mathrm{SDS} \%$ ( $P=$ $0.0167)$ and the MCE risk estimated with CERS after treatment $(P=0.0010)$ were extracted as independent predictors for MCEs in multivariate Cox proportional hazards regression analysis (Table IV).

Comparison of estimated MCE risks before and after treat- ment: MCE risks within 3 years estimated with the CERS after treatment were not different from those estimated before treatment in the patients with MCEs $(13.9 \% \pm 10.8 \%$ versus $14.1 \% \pm 11.1 \% ; P=0.8018)$ but were significantly lower in the patients without MCEs $(9.4 \% \pm 8.0 \%$ versus $11.5 \% \pm$ $8.6 \% ; P<0.0001)$.

MCE risks within 3 years estimated with the CERS in patients having $\geq 5 \%$ ischemic reduction were significantly lower after than before treatment $(8.9 \% \pm 7.9 \%$ versus $12.3 \%$ $\pm 9.5 \% ; P<0.0001)$. In contrast, MCE risks within 3 years estimated with CERS in patients having no $\geq 5 \%$ ischemic reduction were significantly higher after than before treatment $(11.7 \% \pm 8.8 \%$ versus $10.6 \% \pm 7.4 \% ; P=0.0007)$.

Correlation between estimated MCE risks reduction and $\Delta$ SDS\%: Figure 1 shows a positive correlation between the MCE risk reduction (\%) within 3 years estimated with CERS and the $\Delta$ SDS $\%(r=0.6242 ; P<0.001)$. A greater $\Delta$ SDS $\%$ was associated with a greater increase in the MCE risk reduction estimated with the CERS. 


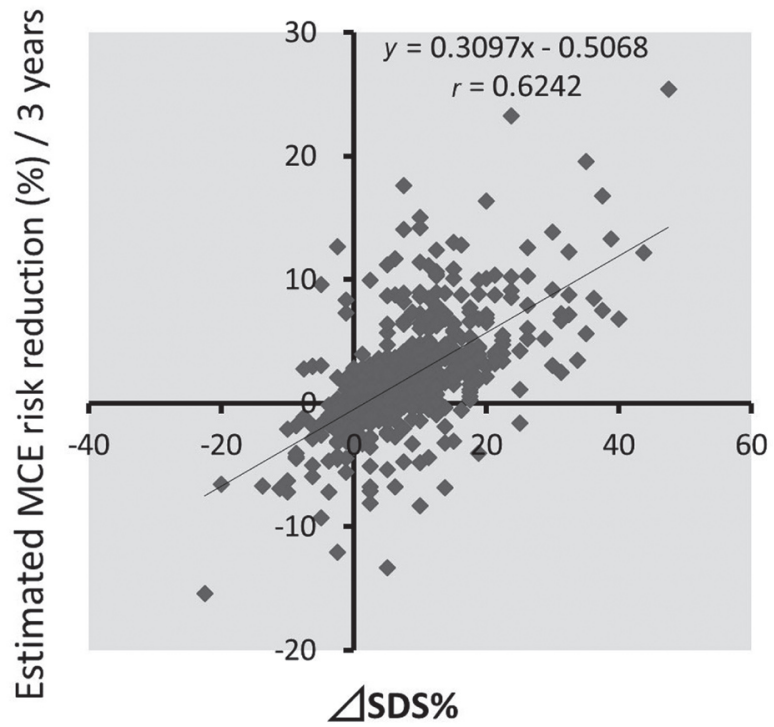

Figure 1. Correlation between MCE risk reduction within 3 years estimated with CERS and $\triangle$ SDS\%. CERS indicates cardiac event risk score; $\Delta \mathrm{SDS} \%$, percent change in summed difference score; and $r$, correlation coefficient.

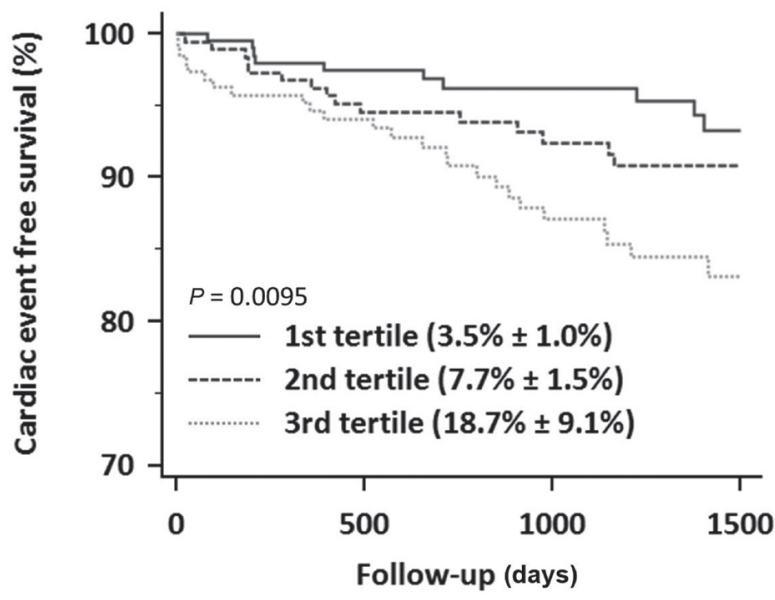

Figure 2. Kaplan-Meier curves for the actual MCE risks in patients divided into 3 categories with tertile. The data shown in parentheses indicate MCE risks estimated with the CERS. MCE indicates major cardiac event; and CERS, cardiac event risk score.

Comparison of MCE risks estimated after treatment with actual occurrences of MCEs: The patients were divided into 3 categories on the basis of the MCE risks estimated with the CERS. Figure 2 illustrates Kaplan-Meier curves for the actual MCE risks and Figure 3 shows a comparison between the actual MCE risks and the MCE risk estimated with CERS in the 3 categories. The proportions of patients who actually experienced MCEs were $5.1 \%(10 / 198)$ in the 1 st tertile, $8.1 \%$ $(15 / 186)$ in the 2 nd tertile, and $13.3 \%$ (25/188) in the 3rd tertile. The tertile division was significant stratification $(P=$ 0.0095). The actual MCE risk was significantly higher in the 3rd tertile than in the 1st tertile $(P=0.0082)$ and was also significantly different between the 3 categories $(P=0.0042)$.

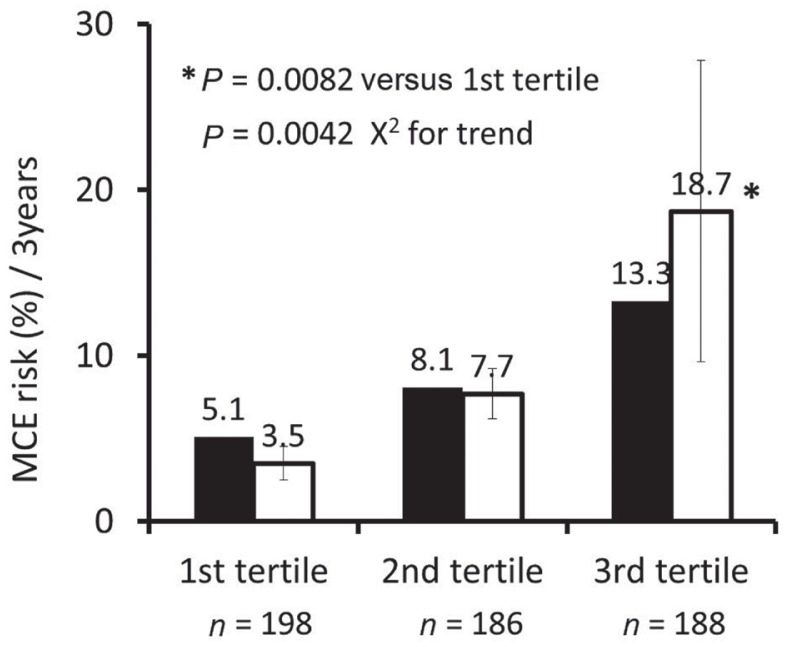

Figure 3. Comparison between the actual MCE risks (closed column) and the MCE risks estimated with the CERS (open column) in patients divided into 3 categories with tertile. MCE indicates major cardiac event; and CERS, cardiac event risk score.

MCE risks within 3 years estimated with the CERS after treatment, which were $3.5 \% \pm 1.0 \%$ for the 1 st tertile, $7.7 \% \pm 1.5 \%$ for the 2nd tertile, and $18.7 \% \pm 9.1 \%$ for 3rd tertile, almost included each of the actual MCE risks.

Typical images in a patient having good prognosis: Figure 4 illustrates serial polar maps obtained from a typical patient having good prognosis after undergoing PCI.

The patient was a 69 -year-old male with stable effort angina. The first SPECT showed extensive ischemia in the region of the left anterior descending artery (LAD) in October 2009. He underwent CAG, which revealed $90 \%$ stenosis in the proximal region of the LAD, and then PCI for the stenosis in the LAD. No ischemia was detected in the LAD region by the second SPECT in April 2010. Thereafter, he experienced no cardiac events and had a good prognosis. His MCE risk within 3 years estimated with CERS was $31.4 \%$ before PCI but definitely decreased to $6.0 \%$ after PCI on the basis of his data: eGFR was $63 \mathrm{~mL} /$ minute $/ 1.73 \mathrm{~m}^{2}$; DM was present; and SSS was 38 and zero before and after PCI, respectively.

\section{Discussion}

This is the first report demonstrating the prognostic value of a CERS predicting the risk of MCEs (except severe heart failure) within 3 years in Japanese patients with CAD, which was evaluated on the basis of the results from a retrospective single-center nuclear cardiology study. This report has considerable significance because the prognostic value of the CERS was verified on the basis of comparison between MCE risks estimated with the CERS and the actual occurrences of MCEs in patients who were not involved in the previous study on preparation of the CERS. MCE risks estimated with the CERS after treatment were selected as an independent predictor based on the results of the multivariate analysis, and were consistent with the actual occurrences of MCEs demonstrated by tertiledivided Kaplan-Meier analysis and the chi-square for trend 

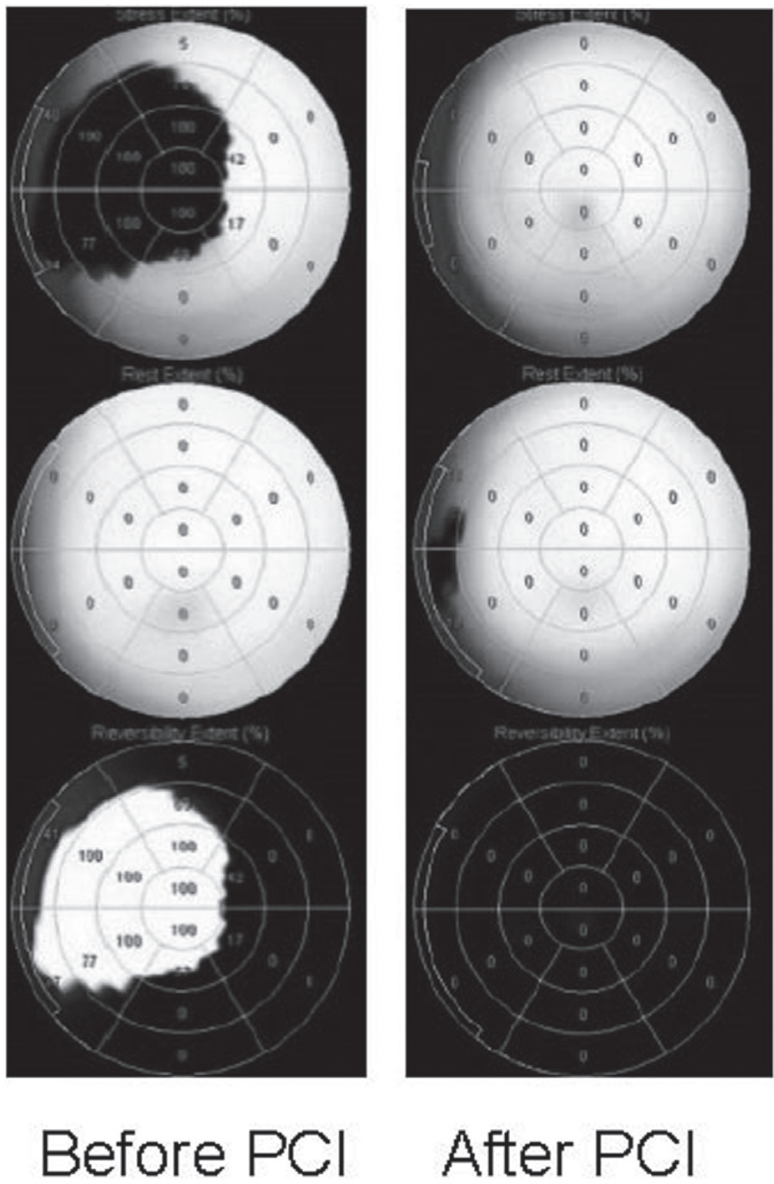

Figure 4. Serial polar maps of stress (upper), rest (middle), and ischemic (bottom) QPS images in a typical patient having good prognosis after undergoing PCI. QPS indicates quantitative perfusion single photon emission computed tomography; and PCI, percutaneous coronary intervention.

test. The results of this verification indicate that the CERS has a high significance for prediction of MCE risks and is a useful clinical tool for a prognosis of patients with CAD.

In addition to the MCE risks estimated with the CERS after treatment, the multivariate analysis extracted $\triangle \mathrm{SDS} \%$, which is an index for improvement of ischemia, as an independent predictor. Furthermore, the greater improvement of ischemia was associated with a greater increase in the MCE risk reduction estimated with the CERS. The MCE risks within 3 years were significantly lower after than before treatment in the patients having at least $5 \%$ ischemic reduction while they were significantly higher after than before treatment in the patients having less than $5 \%$ ischemic reduction. The results of the nuclear substudy of the COURAGE trial ${ }^{19)}$ also showed that patients having at least $5 \%$ ischemic reduction after treatment could have a significantly better prognosis in comparison with those having less than $5 \%$ ischemic reduction. The extent of ischemic reduction after treatment is considered to change or affect the prognosis in patients with CAD. We previously reported a significantly preferable prognosis in patients having at least $5 \%$ ischemic reduction in comparison with patients having less than $5 \%$ ischemic reduction. ${ }^{20)}$ The significance of ischemic reduction for predicting the prognosis was endorsed by the results obtained from the present study.

The Heart Risk Table reported by Nakajima, et $a l^{9)}$ is another CERS predicting the risk of MCEs on the basis of data regarding age, DM, LVEF, SSS categories, and eGFR in Japanese patients with CAD. This is similar to our CERS with respect to the use of patient demographic and baseline characteristics as well as data on ischemic quantification obtained from nuclear cardiology. However, their MCEs included severe heart failure; of the total of 116 MCEs, $64(55.2 \%)$ were severe heart failure requiring hospitalization. Therefore, the Heart Risk Table may be a suitable model for predicting the risk of severe heart failure, but might not be applicable to the prediction of risk of acute coronary syndrome. Furthermore, the prognostic value of the Heart Risk Table is unclear because there is no practical report regarding the predictive accuracy of MCE risks estimated with the Table and the risk reduction after treatment.

Many reports have indicated that LVEF is an important predictor of cardiac events. ${ }^{5,21)} \mathrm{LVEF}$ is one of the predictors useful in the estimation of MCE risks with the Heart Risk Table. However, in a previous study, ${ }^{10)}$ we found that LVEF was an independent significant predictor of cardiac death but not of MCEs. Therefore, we have separately prepared two different equations to predict the risks of MCEs and cardiac death. The discrepancy between our equations and the Heart Risk Table may be based on the definition of whether to include severe heart failure in MCEs. In our study, severe heart failure was not included in the MCEs. We consider LVEF or LVEDV to be a significant predictor of cardiac death and severe heart failure. Actually, the correlations were moderate between SSS and stress LVEF ( $r=0.6331)$ or LVEDV $(r=0.5457)$ after treatment in this study. The equation incorporating LVEF for prediction of cardiac death will be verified in a future study because the aim of the present study was to verify the equation of MCE risks and also the number of patients with cardiac death observed was too small $(n=16)$ in this study to verify the equation of cardiac death risks.

Determination of a therapeutic strategy and the prediction of prognosis in patients with CAD could also be performed with fractional flow reserve, which is a physiological ischemic variable, measured before and after revascularization. ${ }^{22-24}$ However, it is difficult to measure the fractional flow reserve in all stenotic vessels and measurement entails the risks associated with an invasive operation. Therefore, the CERS obtained from nuclear cardiology, which is a non-invasive operation, are preferable to determine a therapeutic strategy for improving the prognosis in Japanese patients with CAD.

Study limitations: Our CERS may involve selection bias because it was created on the basis of data from a retrospective single-center investigation. Also, the timings of treatment and evaluation of its effect to examine MCE risk reduction varied from patients to patients because it was a retrospective study. However, the CERS is a useful index with which to predict the risk of MCEs, with the exception of severe heart failure, because they cannot be predicted with the version of the Heart Risk Table that is currently available in Japan. Because this study verified the prognostic value of the CERS as a unique risk equation for predicting coronary events in Japanese patients with CAD, CERS has significance clinically. In addition, this CERS is easily calculated with general spreadsheet software and provides the rate of MCEs within 3 years. Therefore, 
we believe the CERS will be confirmed by other institutes.

Most of the MCEs were UAP in this study. The most frequent cause for UAP was identified as progression of residual non-culprit stenosis, which is considered to be possibly associated with a low frequency of statin treatment.

Also, radiation exposure from cardiac imaging has become an important topic recently. The level of radiation exposure from the ${ }^{201} \mathrm{Tl}+{ }^{99 \mathrm{~m}} \mathrm{Tc}$-tetrofosmin dual isotope SPECT received by the patients in this study was nearly $30 \mathrm{mSv}$, which was higher than that from ${ }^{99 \mathrm{~m}} \mathrm{Tc}$-tetrofosmin rest-stress SPECT $(8.6 \mathrm{mSv})^{25,26)}$ However, we used this protocol because the patients were not restricted for a long time due to the short procedural length and the patients were informed of the imaging results immediately after completion of the procedure. Our risk score may be applicable to the ${ }^{99 \mathrm{~m}} \mathrm{Tc}$-tetrofosmin rest-stress SPECT protocol because the prediction of prognosis or diagnostic accuracy with ${ }^{201} \mathrm{TI}$ is generally similar to that with ${ }^{99 \mathrm{~m}}$ Tc-tetrofosmin. ${ }^{27)}$

The CERS after treatment is a valuable formula for predicting prognosis in Japanese patients with CAD.

\section{Disclosure}

Conflict of interest: All authors declare that they have no conflict of interest.

\section{ACKNOWLedgments}

We appreciate Miss Yukiko Inoue for assisting with the collection and analysis of the data.

\section{REFERENCES}

1. Wilson PW, D'Agostino RB, Levy D, Belanger AM, Silbershatz H, Kannel WB. Prediction of coronary heart disease using risk factor categories. Circulation 1998; 97: 1837-47.

2. Goff DC Jr, Lloyd-Jones DM, Bennett G, et al; American College of Cardiology/American Heart Association Task Force on Practice Guidelines. 2013 ACC/AHA Guideline on the assessment of cardiovascular risk: a report of the American College of Cardiology/ American Heart Association Task Force on Practice Guidelines. Circulation 2014; 129: S49-73.

3. Hachamovitch R, Berman DS, Shaw LJ, et al. Incremental prognostic value of myocardial perfusion single photon emission computed tomography for the prediction of cardiac death: differential stratification for risk of cardiac death and myocardial infarction. Circulation 1998; 97: 535-43.

4. Hachamovitch R, Hayes SW, Friedman JD, Cohen I, Berman DS Comparison of the short-term survival benefit associated with revascularization compared with medical therapy in patients with no prior coronary artery disease undergoing stress myocardial perfusion single photon emission computed tomography. Circulation 2003; 107: 2900-7.

5. Nishimura T, Nakajima K, Kusuoka H, Yamashina A, Nishimura S Prognostic study of risk stratification among Japanese patients with ischemic heart disease using gated myocardial perfusion SPECT: J-ACCESS study. Eur J Nucl Med Mol Imaging 2008; 35: $319-28$

6. Nakajima K, Yamasaki Y, Kusuoka H, et al. Cardiovascular events in Japanese asymptomatic patients with type 2 diabetes: a 1-year interim report of a J-ACCESS 2 investigation using myocardial perfusion imaging. Eur J Nucl Med Mol imaging 2009; 36: 204957.

7. Joki N, Hase H, Kawano Y, et al. Myocardial perfusion imaging for predicting cardiac events in Japanese patients with advanced chronic kidney disease: 1-year interim report of the J-ACCESS 3 investigation. Eur J Nucl Med Mol Imaging 2014; 41: 1701-9.

8. Matsumoto N, Sato Y, Suzuki Y, et al. Prognostic value of myocardial perfusion single-photon emission computed tomography for the prediction of future cardiac events in a Japanese population: a middle-term follow-up study. Circ J 2007; 71: 1580-5.

9. Nakajima K, Matsuo S, Okuyama C, et al. Cardiac event risk in Japanese subjects estimated using gated myocardial perfusion imaging, in conjunction with diabetes mellitus and chronic kidney disease. Circ J 2012; 76: 168-75.

10. Yoda S, Nakanishi K, Tano A, et al. Major cardiac event risk scores estimated with gated myocardial perfusion imaging in Japanese patients with coronary artery disease. J Cardiol 2016; 67: 6470.

11. Yoda S, Nakanishi K, Tano A, et al. Risk stratification of cardiovascular events in patients at all stages of chronic kidney disease using myocardial perfusion SPECT. J Cardiol 2012; 60: 377-82.

12. Berman DS, Kiat H, Friedman JD, et al. Separate acquisition rest thallium-201/stress technetium-99m sestamibi dual-isotope myocardial perfusion single-photon emission computed tomography: a clinical validation study. J Am Coll Cardiol 1993; 22: 1455-64.

13. Yoda S, Sato Y, Matsumoto N, et al. Incremental value of regional wall motion analysis immediately after exercise for the detection of single-vessel coronary artery disease - Study by separate acquisition, dual-isotope ECG-gated single-photon emission computed tomography. Circ J 2005; 69: 301-5.

14. Yoda S, Nakanishi K, Tano A, et al. Diagnostic value of automated quantification of nuclear cardiology in Japanese patients with single vessel coronary artery disease: comparison between Japanese and American normal databases. J Cardiol 2013; 62: 224-9.

15. Yoda S, Nakanishi K, Tano A, et al. Validation of automated quantification of nuclear cardiology in Japanese patients using total perfusion deficits: comparison with visual assessment. J Cardiol 2013; 63: 350-7.

16. Yoda S, Nakanishi K, Tano A, et al. Usefulness of automated assessment of nuclear cardiology for prediction of major cardiac events in Japanese patients with known or suspected coronary artery disease: comparison with conventional visual assessment in a large-scale prognostic study. J Cardiol 2014; 64: 395-400.

17. Germano G, Kiat H, Kavanagh PB, et al. Automatic quantification of ejection fraction from gated myocardial perfusion SPECT. J Nucl Med 1995; 36: 2138-47.

18. Matsuo $\mathrm{S}$, Imai $\mathrm{E}$, Horio $\mathrm{M}$, et al. Revised equations for estimated GFR from serum creatinine in Japan. Am J Kidney Dis 2009; 53: 982-92.

19. Shaw LJ, Berman DS, Maron DJ, et al. Optimal medical therapy with or without percutaneous coronary intervention to reduce ischemic burden: results from the clinical outcomes utilizing revascularization and aggressive drug evaluation (COURAGE) trial nuclear substudy. Circulation 2008; 117: 1283-91.

20. Hori Y, Yoda S, Nakanishi K, et al. Myocardial ischemic reduction evidenced by gated myocardial perfusion imaging after treatment results in good prognosis in patients with coronary artery disease. J Cardiol 2015; 65: 278-84.

21. Sharir T, Germano G, Kavanagh PB, et al. Incremental prognostic value of post-stress left ventricular ejection fraction and volume by gated myocardial perfusion single photon emission computed tomography. Circulation 1999; 100: 1035-42.

22. Tonino PA, Fearon WF, De Bruyne B, et al. Angiographic versus functional severity of coronary artery stenoses in the FAME study fractional flow reserve versus angiography in multivessel evaluation. J Am Coll Cardiol 2010; 55: 2816-21.

23. De Bruyne B, Pijls NH, Kalesan B, et al. Fractional flow reserveguided PCI versus medical therapy in stable coronary disease. $\mathrm{N}$ Eng J Med 2012; 367: 991-1001.

24. Pijls NH, van Schaardenburgh P, Manoharan G, et al. Percutane- 
ous coronary intervention of functionally nonsignificant stenosis 5-year follow-up of the DEFER study. J Am Coll Cardiol 2007; 49: 2105-11.

25. Einstein AJ, Moser KW, Thompson RC, Cerqueira MD, Henzlova MJ. Radiation dose to patients from cardiac diagnostic imaging. Circulation 2007; 116: 1290-305. (Review)

26. ICRP. Radiation dose to patients from radiopharmaceuticals. Addendum 3 to ICRP Publication 53. ICRP Publication 106. Ap- proved by the Commission in October 2007. Ann ICRP 2008; 38 : $1-197$.

27. Gibbons RJ, Chatterjee K, Daley J, et al. ACC/AHA/ACP-ASIM guidelines for the management of patients with chronic stable angina: a report of the American College of Cardiology/American Heart Association Task Force on Practice Guidelines (Committee on Management of Patients With Chronic Stable Angina). J Am Coll Cardiol 1999; 33: 2092-197. 